\title{
Introduction to the special issue for the ECML PKDD 2019 journal track
}

\author{
Karsten Borgwardt ${ }^{1}$ - Po-Ling Loh $^{2}$ - Evimaria Terzi ${ }^{3}$. Antti Ukkonen ${ }^{4}$
}

Received: 1 July 2019 / Accepted: 5 July 2019 / Published online: 20 July 2019

(c) The Author(s), under exclusive licence to Springer Science+Business Media LLC, part of Springer Nature 2019

This special issue contains papers accepted to Data Mining and Knowledge Discovery as part of the journal track of ECML PKDD 2019. This continues the tradition that began at ECML PKDD 2013, and has been a popular part of the ECML PKDD conference series ever since. By submitting their paper to the journal track, authors can both obtain a journal publication, as well as enjoy the benefit of giving a presentation about their work in a conference setting. This also means, however, that the type of papers eligible for submission to the journal track has been restricted to original research articles, and thus survey papers as well as extended versions of already published conference papers are not allowed.

Since its inception seven years ago, the ECML PKDD journal track has been organised jointly with Springer's Data Mining and Knowledge Discovery and Machine Learning journals. The ECML PKDD 2019 journal track was organized around five submission deadlines, distributed approximately evenly in 5-week intervals between August 13, 2018 and January 21, 2019. Authors could choose which of the two journals they would like their paper to appear in; the ECML PKDD journal track chairs serve as the guest editors in both journals. For the most part, papers submitted to the journal track were reviewed by members of a Guest Editorial Board after a round of

\section{Responsible editor: Johannes Fürnkranz.}

Antti Ukkonen

antti.ukkonen@helsinki.fi

Karsten Borgwardt

karstenb@ethz.ch

Po-Ling Loh

ploh@stat.wisc.edu

Evimaria Terzi

evimaria@bu.edu

1 Department of Biosystems Science and Engineering, ETH Zürich, Zurich, Switzerland

2 Department of Statistics, University of Wisconsin - Madison, Madison, WI, USA

3 Computer Science Department, Boston University, Boston, MA, USA

4 Department of Computer Science, University of Helsinki, Helsinki, Finland 
bidding for papers. A number of reviews were also obtained from other specialists. Papers accepted to this year's journal track will be presented by their authors at ECML PKDD 2019 in Würzburg, Germany from September 16 to 20, 2019.

In total, 70 papers were submitted to Data Mining and Knowledge Discovery. Of these, for five manuscripts, the reviewing process was completed in time for the paper to be included in this special issue. This issue also contains six submissions to the journal track for ECML PKDD 2018 that were still under review when the associated special issue was finalized. The editors of these papers were the journal track chairs of ECML PKDD 2018: Jesse Davis, Elisa Fromont, Derek Greene, and Björn Bringmann.

Many individuals have contributed to the success of the journal track. First, we would like to thank all authors who submitted papers. Secondly, we would like to thank the members of our Guest Editorial Board and all other reviewers who provided timely and high-quality reviews. We will honor outstanding reviewing service to this special issue through Reviewer Awards, whose winners will be announced on the ECML PKDD 2019 website. We would also like to extend a very special thanks to Katharina Heinrich (ETH Zürich) who carefully coordinated the bidding, reviewing and editing efforts, allowing the journal track chairs to focus on the scientific content. Thirdly, we acknowledge the help and kind support of Johannes Fürnkranz (Editorin-Chief of Data Mining and Knowledge Discovery), Peter Flach (Editor-in-Chief of Machine Learning), and Melissa Fearon (Senior Editor of Springer responsible for these journals). Finally, we would like to extend our appreciation to the ECML PKDD 2019 general chairs and to last year's journal track chairs for their valuable advice.

The papers in this special issue cover a range of topics within data mining, including representation learning, pattern mining, density estimation, and network analysis. We hope that the papers are enjoyable and interesting, and that they will inspire readers with their own research!

Publisher's Note Springer Nature remains neutral with regard to jurisdictional claims in published maps and institutional affiliations. 